\title{
Do assembly bonus effects occur on disjunctive tasks? A test of Steiner's theory
}

\author{
MARVIN E. SHAW and NANCY ASHTON \\ University of Florida, Gainesville, Florida $\$ 2611$
}

\begin{abstract}
Two experiments were run to test Steiner's hypothesis that actual group performance on disjunctive tasks is equal to potential group performance (based upon the individual competencies of group members), minus losses due to faulty group processes. On no task did groups perform significantly worse than predicted, and on one of the four tasks groups performed significantly better than predicted from individual performance. It was concluded that actual group performance on disjunctive tasks is equal to potential group performance, minus losses due to faulty group processes, plus assembly bonus effects.
\end{abstract}

The question of whether the assembly of individuals into groups facilitates or impedes problem solving effectiveness has been a controversial issue for many years, but a definitive resolution has not been achieved. Early investigations comparing individual and group performance (e.g., Shaw, 1932; Watson, 1928) generally revealed that groups produced more and better solutions, but at the expense of increased man-hours. More recent theoretical and experimental work (e.g., Steiner, 1972; Tuckman \& Lorge, 1962) indicates that the kind of task is probably the most significant determinant of the relative effectiveness of groups and individuals.

Perhaps the most systematic attempt to relate task characteristics to individual and group performance is Steiner's (1972) analysis. He proposed that tasks may be classified as additive, conjunctive, or disjunctive. A task is said to be additive when the requirements of the task are such that success of the group depends upon a summation of individual products. Group performance on additive tasks is presumed to be a function of the average competence of the individuals composing the group. A conjunctive task is one that requires that each group member perform the task. Theoretically, group performance on conjunctive tasks depends upon the least competent group member. A disjunctive task is one that requires an either-or decision or a choice among alternatives. According to Steiner's theory, group performance depends upon the most competent person in the group. In each instance, it is theoretically possible to predict group performance from knowledge of individual performance. In this paper, we shall be concerned with group performance on disjunctive tasks.

On the assumption that a group can complete a disjunctive task if at least one member of the group can complete it, the problem of predicting the proportion of groups that can complete any given task becomes one of determining the probability that any given group of $n$

Requests for reprints should be sent to Marvin E. Shaw, Department of Psychology, University of Florida, Gainesville, Florida 32611. size will contain at least one person who is capable of completing the task. Steiner (1972) noted that the percentage of groups of $n$ size containing at least one person who can complete the task is $100\left(1-Q^{n}\right)$, where $\mathrm{Q}$ is the proportion of persons in the population who cannot perform the task. This percentage, therefore, represents the percentage of groups that should perform the task successfully, if there are no losses in potential due to faulty group processes. According to Steiner, since faulty group processes usually occur, actual group productivity is equal to potential productivity minus losses due to faulty group processes.

These proposals are interesting, but there is no provision for any positive contributions resulting from efficient group interaction. Steiner (1972) apparently believes that group process, if it has any effect at all, can only impair group effectiveness. However, it is certainly possible that the inputs of one group member can stimulate another group member, eliciting responses that would not occur if the individual were alone. Still others in the group may be stimulated to produce new ideas and innovative responses, so that the net outcome is an increase in the effectiveness of the group. Such an "assembly bonus effect" has been proposed by Collins and Guetzkow (1964), and findings consistent with this interpretation have been reported with respect to additive tasks (e.g., Yuker, 1955). Although this effect apparently has not been demonstrated for disjunctive tasks, it is theoretically possible that assembly bonus effects occur on this type of task as well. The primary purpose of the experiments reported here was to examine this possibility.

In addition, the probability that assembly bonus effects will occur may be expected to vary with the proportion of persons in the group who are capable of performing the group task. Hence, the more difficult the task (that is, the smaller the proportion of individuals who can perform the task working alone), the more likely it is that assembly bonus effects will occur. Therefore, we should expect the ratio of actual productivity to potential productivity to increase with increasing task 
difficulty. A secondary purpose of this experiment was to test this expectation.

\section{EXPERIMENT I}

\section{Method}

The experimental design for this study was a $2 \times 2 \times 2 \times 2$ mixed factorial, involving individual vs. group problem solving, male and female subjects, two levels of task difficulty, and two task orders. Task difficulty involved repeated measures: Individuals and groups attempted both easy and difficult tasks in counterbalanced order.

Subjects. The subjects for this experiment were $\mathbf{4 0}$ males and 40 females drawn from undergraduate courses in psychology. They were randomly assigned to either the individual or the group situation, so that 10 males and 10 females attempted the two tasks working alone and 10 three-person male groups and 10 three-person female groups attempted the two tasks working together.

Tasks. The tasks were crossword puzzles selected to vary in difficulty. In each instance, the goal was to identify a single entry in the puzzle, e.g., 38 across, 42 down, etc. This task is similar to the horse-trading problem cited by Steiner (1972) as an example of a disjunctive task. For each task there is a finite number of words that fit the number of spaces to be filled. Successful completion of the task requires identification of the one word among the set of possible words that fits the clue provided for the space in question. Therefore, this task is disjunctive, according to Steiner's definition. One of the tasks was selected to be relatively easy for individuals working alone, based on pretest data, while the other was selected to be relatively difficult for an individual working alone.

Procedure. Subjects were greeted at the experimental room and instructions were read to them by the experimenter. Pilot studies had indicated that relatively few students could be expected to be experienced in solving crossword puzzles; therefore, both individuals and groups were given detailed instructions concerning the task. They were also told that they would be attempting two tasks and would be allowed $30 \mathrm{~min}$ to complete each task.

Groups were seated at a round table with a copy of the task before them. Individuals sat in separate cubicles. For each group and each individual, a record was kept of whether the task was successfully completed, how long it took to complete it, and how many wrong answers were submitted to the experimenter before the correct answer was submitted or the $30 \mathrm{~min}$ expired. At the end of the experiment, subjects filled out a short questionnaire probing their reactions to the tasks and how much experience they had had with crossword puzzles.

\section{Results}

Before examining major findings, it should be noted that performance data (Table 1) verified that tasks differed in difficulty, as planned. Also, data from the postexperimental questionnaire revealed that subjects,

Table 1

Proportion of Individuals and Groups Successfully Completing Tasks in Experiment I

\begin{tabular}{|c|c|c|c|c|}
\hline & \multirow[b]{2}{*}{ Individuals } & \multicolumn{2}{|c|}{ Groups } & \multirow{2}{*}{$\frac{\text { Ratio }}{\text { Obs/Pred }}$} \\
\hline & & Observed & Predicted & \\
\hline $\begin{array}{l}\text { Task A } \\
\text { Task B }\end{array}$ & $\begin{array}{l}.55 \\
.20\end{array}$ & $\begin{array}{l}.80 \\
.45\end{array}$ & $\begin{array}{l}.91^{*} \\
.49 * *\end{array}$ & $\begin{array}{l}.88 \\
.92\end{array}$ \\
\hline
\end{tabular}

${ }^{*} z$ observed $v s$. predicted $=1.38 ; p<.20$

${ }^{* *} z$ observed $v$ s. predicted $=.36 ; p<.72$ as expected, reported only limited experience with crossword puzzles, but the degree of such experience was not significantly different for individuals and groups $[F(1,25)=1.77, p<.20]$.

The major concern of this study was the relationship between group performance predicted on the basis of individual performance and actual (observed) group performance. Since there was no significant difference in proportion of individuals and groups completing tasks as a function of sex of subjects or task order, these variables were ignored in the analysis of data.

Predicted group performance was computed and the significance of the difference between observed and predicted group performance was tested by the $\mathrm{z}$ statistic, using the formula suggested by Bruning and Kintz (1968).

Table 1 gives the relevant data. First, it can be seen that there was no significant difference between observed and predicted group performance on either task. In fact, observed performance closely approximated predicted performance. As in Steiner's (1972) analysis, it is surprising that so little was lost through faulty group processes, assuming that no assembly bonus effects occurred.

It may also be noted in Table 1 that the ratio of observed performance to predicted performance was greater for the more difficult task (Task B), as expected. More will be said about this after the results of Experiment II are presented.

\section{EXPERIMENT II}

\section{Method}

Experiment II was essentially a replication of Experiment I, using more difficult tasks. The design was a $2 \times 2 \times 2$ factorial, with individuals vs. groups, two levels of task difficulty, and two task orders. Ten individuals and 10 groups of three persons each attempted two crossword tasks similar to those used in Experiment I. All individuals and groups attempted both tasks, in counterbalanced order. All subjects were male college students.

\section{Results}

Again, primary interest was in the difference between predicted and observed group performance. Since the proportions of individuals and groups completing tasks did not differ significantly with respect to task order, this variable was not considered in this analysis. The relevant data from Experiment II are given in Table 2.

Table 2

Proportion of Individuals and Groups Successfully Completing Tasks in Experiment II

\begin{tabular}{|c|c|c|c|c|}
\hline & \multirow[b]{2}{*}{ Individuals } & \multicolumn{2}{|c|}{ Groups } & \multirow{2}{*}{$\frac{\text { Ratio }}{\text { Obs/Pred }}$} \\
\hline & & Observed & Predicted & \\
\hline Task A & .20 & .50 & $.49^{*}$ & 1.02 \\
\hline Task B & .10 & .70 & $.27 * *$ & 2.59 \\
\hline
\end{tabular}

${ }^{*} z$ observed $v$. predicted $=.06 ; p<.95$

$* * z$ observed $v$ s. predicted $=3.00 ; p<.01$ 
No significant difference was found between observed and predicted performance on Task A; however, groups performed significantly better on Task B than predicted on the basis of individual performance $(\mathrm{z}=3.00$, $\mathrm{p}<.01)$. This finding is consistent with the hypothesis that assembly bonus effects may occur in groups and contrary to Steiner's prediction that group performance will never exceed, except by chance, the potential expected on the basis of the most competent group member.

It may also be noted in Table 2 that, again, the ratio of observed to predicted group performance was greater for the more difficult task. Considering the four tasks from both experiments, the rank order correlation between task difficulty (as measured by time to solve and number of completions) and the ratio of observed to predicted group performance is .95 (standard error $=.06)$.

\section{DISCUSSION}

The data from the two studies reported in this paper provide little support for Steiner's (1972) proposition that group performance on disjunctive tasks depends solely on the most competent group member minus losses due to faulty group process. On no task did groups perform significantly poorer than predicted from individual performance, and on one of four tasks groups performed significantly better than predicted. In other words, on three tasks groups either followed nearly perfect procedures or assembly bonus effects occurred; on the fourth task, assembly bonus effects definitely occurred. The most reasonable interpretation is that assembly bonus effects occurred on all tasks, but were cancelled out by faulty group processes on three of the four tasks. Therefore, the results from these two studies lend support to the proposition that group productivity on disjunctive tasks is equal to potential group productivity (based upon ability of the most competent member), minus losses due to faulty group processes, plus assembly bonus effects.
This conclusion is bolstered by the finding that the ratio of observed to predicted performance varies with task difficulty. It may be argued that the more difficult the task, the more likely that assembly bonus effects will occur. This expectation is based upon the assumption that easy tasks will be completed relatively quickly by the most competent group member, who will have relatively less difficulty getting the solution accepted by other group members. Consequently, there is less need or opportunity for the information and ideas of some group members to stimulate other group members. On the other hand, when the task is difficult, the group will spend more time attempting to complete the task and interpersonal stimulation should be greater.

The data concerning task difficulty and the presumed occurrence of assembly bonus effects cannot be given great weight because of the small number of tasks. Nevertheless, this finding and the findings relative to actual group performance, taken together, provide strong support for the proposition that both faulty group processes and assembly bonus effects contribute to group performance on disjunctive tasks.

\section{REFERENCES}

Bruning, J. L., \& Kintz, B. L. Computational handbook of statistics. Glenview, Ill: Scott, Foresman, 1968.

Collins, E. B., \& Guetzkow, H. A social psychology of group processes for decision-making. New York: Wiley, 1964.

SHAW, M. E. A comparison of individuals and small groups in the rational solution of complex problems. American Journal of Psychology, 1932, 44, 491-504.

STEINER, I. D. Group processes and productivity. New York: Academic Press, 1972.

TUCKMAN, J., \& LoRGE, I. Individual ability as a determinant of group superiority. Human Relations, 1962, 15, 45-51.

WATson, G. B. Do groups think more effectively than individuals? Journal of Abnormal and Social Psychology, 1928, 23, 328-336.

YUKER, H. E. Group atmosphere and memory. Journal of Abnormal and Social Psychology, 1955, 51, 17-23.

(Received for publication August 10, 1976.) 